\title{
Evolution de l'industrie pétrolière offshore au Mexique
}

\author{
Ma Cristina Garcia-Govea ${ }^{(\mathrm{a})}$, Oscar Valle-Molina ${ }^{(\mathrm{b})}$ \\ (a)Docteur, Instituto Mexicano del Petroleo, Programme de Recherche en Eaux Profondes, \\ Eje Central Lazaro Cardenas No. 152, Col. San Bartolo Atepehuacan \\ Edificio 5, cubiculo 022, CP 07730, Mexico D.F. Mexique, \\ Téléphone: +52 559175 6378, Fax: +52 559175 7225, E-mail : mcgarcia@imp.mx \\ (b)Coordinateur du Programme de Recherche en Eaux Profondes, Instituto Mexicano del Petroleo, \\ Eje Central Lazaro Cardenas No. 152, Col. San Bartolo Atepehuacan, \\ Edificio 28, PB, CP 07730, Mexico D.F. Mexique, \\ Téléphone: +52 559175 7230, Fax: +52 559175 7225, E-mail : ovalle@imp.mx
}

\section{Résumé}

L'industrie des hydrocarbures commence au Mexique en 1900 avec la exploitation du pétrole par des entreprises étrangères. À partir de l'expropriation pétrolière en 1938, l'industrie est gérée par Pétroles Mexicains «Petroleos Mexicanos (Pemex)». En 1949, Pemex développe les premiers travaux exploratoires dans la plate-forme continentale du Golfe du Mexique. Actuellement, Pemex divise le Pays par quatre Régions :

- Région Marine Nord-ouest

- Région Marine Sud-ouest

- Région Nord

- Région Sud

Les deux Régions Marines produisent actuellement $82 \%$ de la production totale des hydrocarbures au Mexique, ça veut dire 2.5 millions de barils par jour.

\section{$\underline{\text { Abstract }}$}

The industry of hydrocarbons begins in Mexico towards 1900 with oilfield in foreigner companies hands.

From the Mexican oil expropriation in 1938, the industry is handled by Petroleos Mexicanos (Pemex). In 1949, Pemex develops the first exploratory works in the continental platform of the Gulf of Mexico. At the moment, Pemex devise the country in four regions:

- North-west Marine Region

- South-west Marine Region

- North Region

- South Region

The two marine regions produce at the moment $82 \%$ of the total hydrocarbon production in Mexico; this means 2.5 millions oil barrels per day.

Mot-clés: courants, hydrocarbures, off-shore, ouragans, pipelines, plate-forme.

Keywords: currents, hurricane, hydrocarbons, offshore, oil pipeline, platform, 


\section{$\underline{\text { 1.Introduction }}$}

L'exploitation des hydrocarbures offshore à niveau mondial commence à la fin du dixneuvième siècle, néanmoins, le Mexique a commencé dans les années soixante-dix du vingtième siècle. Le Mexique est un des principaux producteurs du pétrole au monde (huitième au niveau mondial), ceci représente une partie important de l'économie du pays. Pour cette raison, il est primordial pour le pays, la réalisation de dessins appropriés des pipelines et de plates-formes.

En 1949, Pemex (Petroleos Mexicanos) a développé les premiers travaux exploratoires dans la plate-forme continentale du Golfe du Mexique, face aux Etats de Veracruz et Tabasco. Jusqu'à 1965 commence l'activité offshore avec la découverte des Champs pétroliers «Tiburon» et "Atun» (PEP, 1999). En 1975, les travaux de perforation du puits exploratoire Chac-1 ont été finis, en marquant le début de l'extraction du pétrole brut dans la région (Pemex, 1979). Pour 1979, Pemex comptait avec plusieurs complexes pour l'extraction, production et distribution de hydrocarbures.

Dans la Région Marine Nord-Est, on fait l'exploitation de gisements de pétrole brut et de gaz, où les fluides parcourent plus de 2,500 m depuis le sous sol jusqu'à la surface, (PEP, 1999). La distribution des hydrocarbures dans la Région Marine Nord-Est se réalise à travers un réseau de 1,607 $\mathrm{km}$ de pipelines, où 1,286 $\mathrm{km}$ correspondent aux pipelines marines et 320 aux pipelines terrestres. De la même manière, selon le service proportionné, $782 \mathrm{~km}$ de pipelines correspondent aux oléoducs, $103 \mathrm{~km}$ aux oléo gazoducs, $617 \mathrm{~km}$ aux gazoducs, 8 Kms correspondent aux ducs pour eau et $97 \mathrm{~km}$ sont gazolinoducts, (PEP, 1999). La Région Marine Sud-Est de Pemex, comprend une surface de $352,390 \mathrm{~km}^{2}$. Dans cette Région on fait l'exploitation de gisements de gaz, pétrole brut et pétrole léger. La distribution des hydrocarbures se réalise à travers $901 \mathrm{~km}$ de pipelines, desquels $851 \mathrm{~km}$ sont marins et $50 \mathrm{~km}$ sont terrestres. De la même façon, selon le service proportionné, $471 \mathrm{~km}$ correspondent aux oléoducs, $217 \mathrm{~km}$ aux oléo gazoducs, $185 \mathrm{~km}$ aux gazoducs, $21 \mathrm{~km}$ correspondent aux duc pour l'eau et $6 \mathrm{~km}$ sont gazolinoducts. Actuellement, plus de $50 \%$ de la exploitation des hydrocarbures se réalise dans les installations offshores (PEP, 1999).

\section{La Sonde de Campeche}

La plate-forme continentale dans cette région du Golfe du Mexique possède une pente douce. Le Golfe du Mexique présente un lit marin composé par sable veiné par des rubans de couches de corail. La Sonde de Campeche, elle-même possède une stratigraphie plus compliquée, en étant un mélange de sables et argiles calcaires de diverses densités.

En 1985 le Mexique et le Brésil ont annoncé trois découvertes pétrolières, deux à la Sonde de Campeche et une à Campos Brésil. Cette même année, le Brésil établit un nouveau record dans l'exploitation en eaux profondes, avec un puits exploratoire à $850 \mathrm{~m}$ de profondeur.

\subsection{Classements des plates formes marines au Mexique}

Les plates-formes sont classées conformément à la fonction qui développent ou à la structure avec laquelle elles sont construites (Torres 1998). Le tableau suivant présente ce classement: 


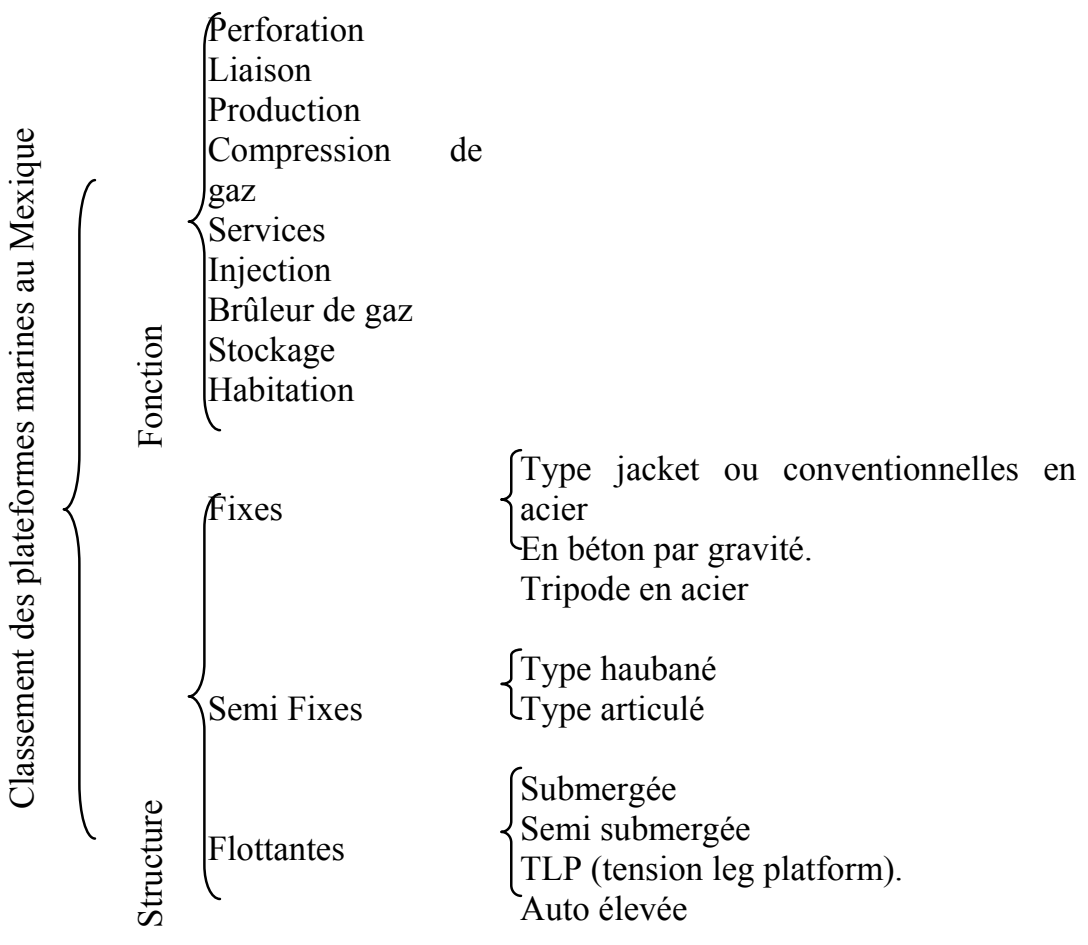

Table 1: classement des plateformes au Golfe du Mexique

\section{Caractérisation de dangers océanographiques et météorologiques}

Conformément aux observations qui ont été effectuées partout dans le monde pour établir la localisation des cyclones tropicaux, actuellement, on a identifié huit zones génératrices d'ouragans, (voir figure 1), desquelles deux d'entre elles affectent directement la totalité des côtes Mexicaines (Soriano, 1999), à savoir :

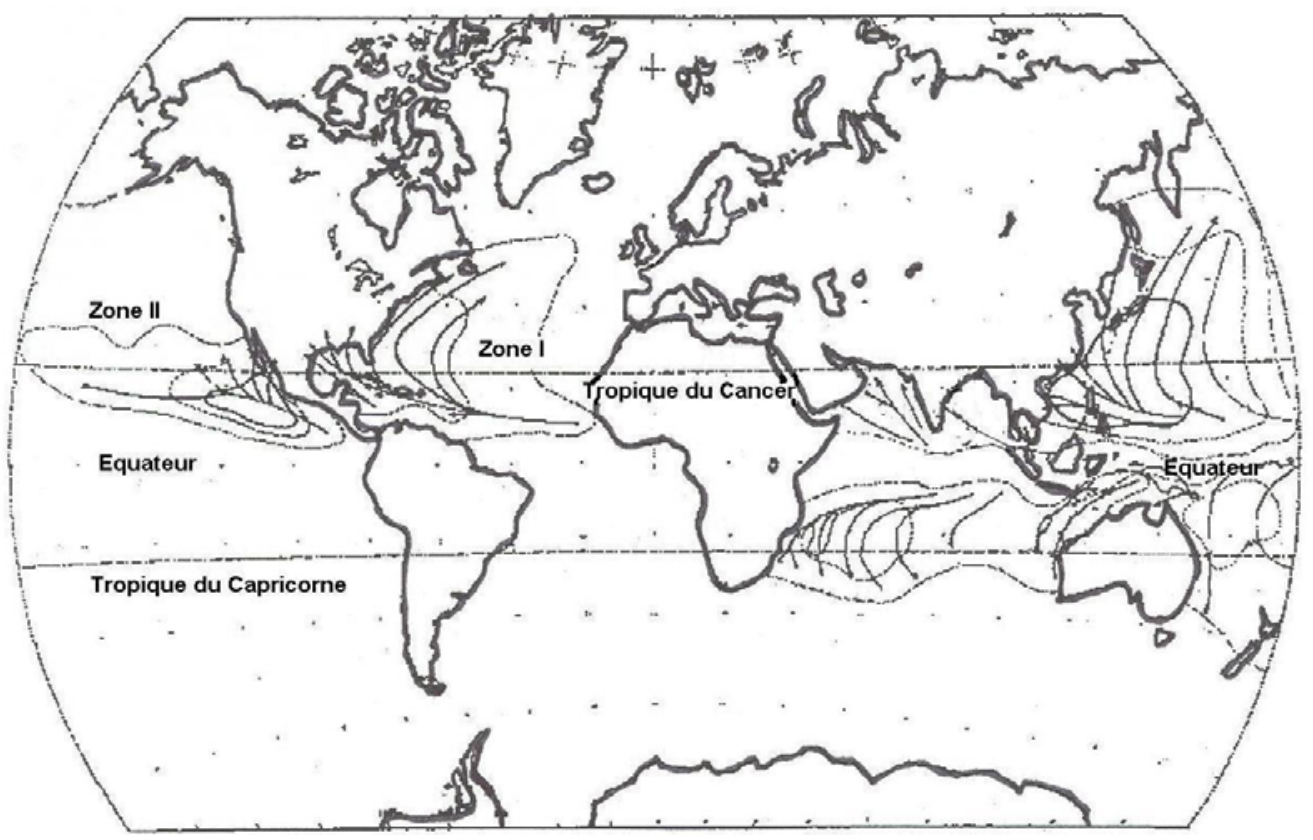

Figure 1: Zones génératrices d'ouragans 
- Zone de cyclons Iou des Indes Occidentales, elle se localise dans l'Atlantique nord et il est précisément ici où commencent les ouragans qui voyagent vers les Caraïbes en atteignant la Péninsule de Yucatan et la Versant du Golfe du Mexique, ainsi que les côtes de l'est et sud est des Etats-Unis.

- Zone de cyclons II: elle se localise dans l'océan Pacifique nord-est, ici se forment les cyclons qui affectent les côtes du Pacifique mexicain. La zone de cyclons de l'océan Pacifique nord-est est une des plus grandes zones génératrices de cyclons au monde.

\subsection{Principaux effets destructif des ouragans aux structures offshore au Mexique}

Le Mexique est plongé géographiquement dans une des zones sujettes au pas des ouragans, les effets perturbateurs de ces phénomènes météorologiques affectent gravement les installations côtières qui sont situées dans le littoral mexicain.

Les ouragans qui affectent les côtes mexicaines du Pacifique et du Golfe du Mexique appartiennent aux zones de générations d'ouragans I et II. Pour leur étude on a établi quatre zones d'origine (Soriano 1999) en ces dernières, les ouragans apparaissent avec un différent degré d'intensité. Ces phénomènes se présentent depuis la dernière semaine de mai et jusqu'au la première moitie d'octobre, parfois au début novembre (figure 2). Les quatre zones d'origine d'ouragans qui affectent les côtes du Pacifique et du Golfe du Mexique sont:

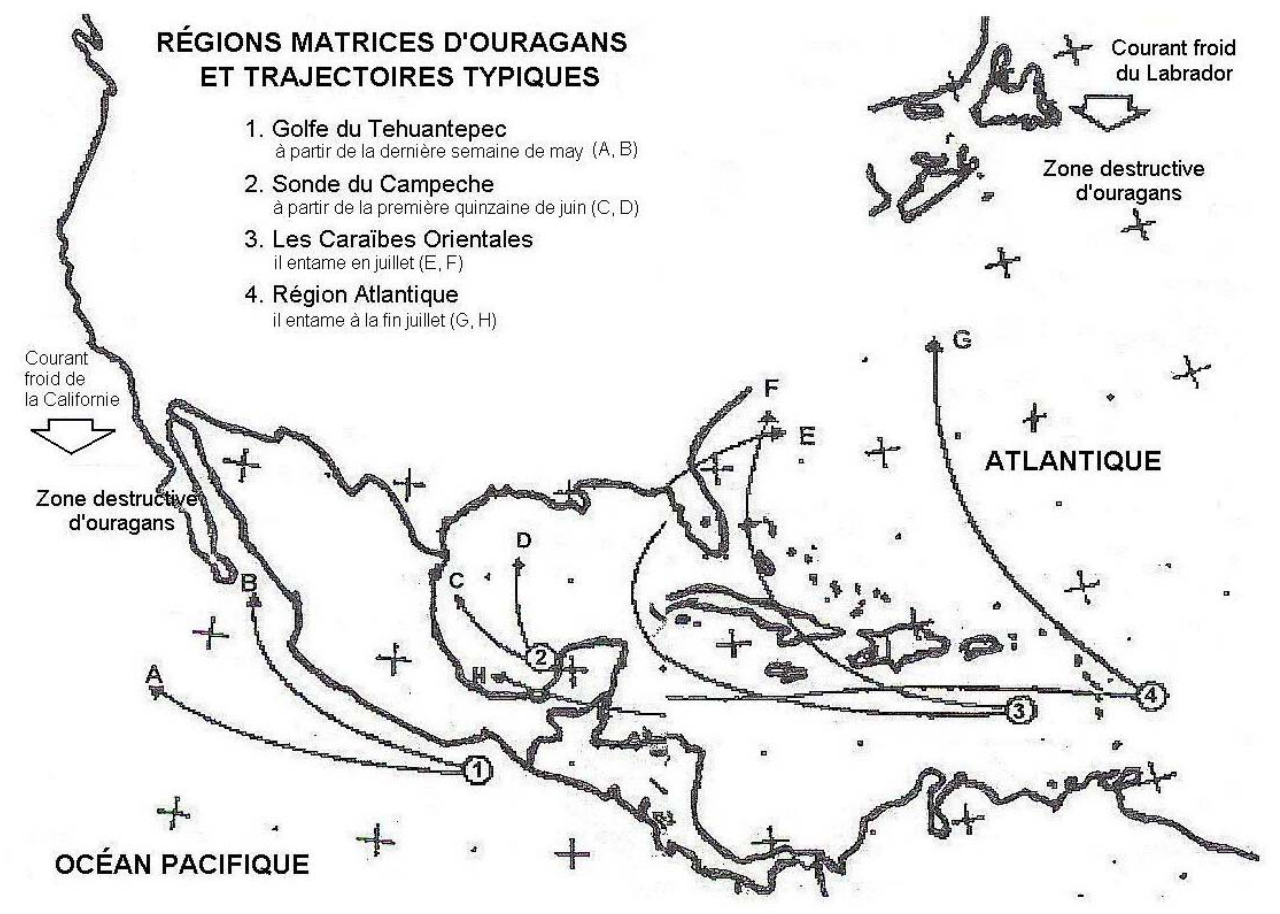

Figure 2: Ouragans qui affectent les côtes mexicaines

- Région Matrice du Pacifique nord-est.

- Région Matrice du Golfe du Mexique

- Région Matrice des Caraïbe

- Région Matrice de l'Atlantique 
Les ouragans qui se génèrent dans la Région Matrice du Pacifique nord-est ont leur origine dans le Golfe de Tehuantepec. La saison entame la dernière semaine de mai, en commençant l'époque de pluies.

Les ouragans qui se forment dans la Région Matrice du Golfe du Mexique ont leur origine dans la Sonde de Campeche et ils entrent en action la première quinzaine de juin en latitude $22^{\circ}$ Nord.

La Zone Matrice des Caraïbes est placée en latitude $13^{\circ}$ Nord, en étant établi au mois de juillet quand le chauffage de la mer a envahi la Région insulaire des Antilles, en se formant des ouragans de grand parcours et de puissance extraordinaire, particulièrement pendant les mois de août, septembre et octobre.

La Zone Matrice de l'Atlantique est placée entre les latitudes $8^{\circ}$ et $12^{\circ}$ Nord, au sud des îles du Cap Vert et les ouragans se produisent fin juillet.

Les Zones Matrices précédemment mentionnées éprouvent de déplacements qui obéissent aux centres de chauffage maritime maximal et à l'interaction entre les courants froids de la Californie et la contre courant équatorial chaud du Pacifique.

Les cyclons extratropicaux se présentent immédiatement après la saison de cyclons. Ce type de phénomènes est connu ave le nom de «Nords». Les Nords se sont produits au début de la saison hivernale, quand le gradient de température de la mer a presque disparu et il y a un équilibre entre la température de la mer et celle de l'air. Le tableau suivant présente les dommages typiques éprouvés par les plates-formes dans la Sonde de Campeche.

\begin{tabular}{|c|c|c|}
\hline Туре & Zone & Description \\
\hline 1 & $\begin{array}{l}\text { Escalier du niveau }+6.096 \mathrm{~m} \text { au niveau } \\
+15.850 \mathrm{~m}\end{array}$ & $\begin{array}{l}\text { Détachements ou déflections } \\
\text { sévères. }\end{array}$ \\
\hline 2 & Balustrades en couloirs du niveau $+6.096 \mathrm{~m}$ & Détachements ou déflections \\
\hline 3 & Grille en couloirs du niveau $+6.096 \mathrm{~m}$ & Détachements en diverses zones. \\
\hline 4 & Escaliers en débarcadère & Détachements ou déflections \\
\hline 5 & Balustrades en débarcadères & Détachements ou déflections \\
\hline 6 & Grille en débarcadères & Détachements en diverses zones \\
\hline 7 & $\begin{array}{l}\text { Plateformes pour l'opération de soupapes } \\
\text { ou structures pour le support d'équipe } \\
\text { mineur situées sous le niveau }+15.850 \mathrm{~m}\end{array}$ & Plis sévères \\
\hline 8 & Pipelines verticaux & $\begin{array}{l}\text { Bosselures et braquages de la } \\
\text { position d'origine }\end{array}$ \\
\hline 9 & $\begin{array}{l}\text { Support des pipelines sous le niveau } \\
+15.850 \mathrm{~m}\end{array}$ & Déflections \\
\hline 10 & Equipes situés sous le niveau $+15.850 \mathrm{~m}$ & Glissements et renversements \\
\hline 11 & $\begin{array}{l}\text { Plaque du système d'étage au niveau } \\
+15.850 \mathrm{~m}\end{array}$ & Déformations \\
\hline 12 & $\begin{array}{l}\text { Poutres du système d'étage au niveau } \\
+15.85 \mathrm{~m}\end{array}$ & $\begin{array}{l}\text { Déformations du patin inférieure } \\
\text { dans les appuis de sous niveaux et } \\
\text { supports des pipelines }\end{array}$ \\
\hline 13 & Poutres principales en élévation $+15.850 \mathrm{~m}$ & $\begin{array}{l}\text { Déformations latérales dans les } \\
\text { patins et les âmes }\end{array}$ \\
\hline
\end{tabular}

Table 2: Type de dommages présentés pour les plates-formes pétrolières du Golfe du Mexique 
La table 3 présente les dommages éprouvés selon le type de plate-forme dans la Région sud-ouest de Pemex dans la Sonda de Campeche.

\begin{tabular}{|c|c|c|c|c|}
\hline $\begin{array}{l}\text { Champ } \\
\text { Pétrolier }\end{array}$ & $\begin{array}{l}\text { Type de } \\
\text { service }\end{array}$ & $\begin{array}{l}\text { Type de } \\
\text { plateforme }\end{array}$ & $\begin{array}{l}\text { Profond } \\
\text { eur } \\
\text { (m) }\end{array}$ & $\begin{array}{l}\text { Type } \\
\text { d'endommagement } \\
\text { présenté (table 2) }\end{array}$ \\
\hline $\begin{array}{l}\text { Abkatum } \\
\text { «A» }\end{array}$ & Habitation & Octopode & 35.80 & $1,2,3,5,6,7$ \\
\hline $\begin{array}{l}\text { Abkatum } \\
\text { «A» }\end{array}$ & $\begin{array}{l}\text { Compressi } \\
\text { on }\end{array}$ & Octopode & 37.80 & $1,2,3,5,6,9$ \\
\hline $\begin{array}{l}\text { Abkatum } \\
\text { «A» }\end{array}$ & $\begin{array}{l}\text { Productio } \\
\mathrm{n}\end{array}$ & Dodécapode & 36.50 & $1,2,3,5,6,7,9,11$ \\
\hline $\begin{array}{l}\text { Abkatum } \\
\text { «A» }\end{array}$ & $\begin{array}{l}\text { Productio } \\
\mathrm{n}\end{array}$ & Octopode & 35.90 & $1,2,3,4,5,6$ \\
\hline $\begin{array}{l}\text { Abkatum } \\
\text { «A» }\end{array}$ & Liaison & Octopode & 37.80 & $1,2,3,5,6,7,9$ \\
\hline $\begin{array}{l}\text { Abkatum } \\
\text { «A» }\end{array}$ & $\begin{array}{l}\text { Perforatio } \\
n\end{array}$ & Octopode & 36.10 & $1,2,3,5,6,7,9$ \\
\hline $\begin{array}{l}\text { Abkatum } \\
\text { «A» }\end{array}$ & D’appui & Tétrapode & 36.00 & $1,2,3,4,5,6$ \\
\hline $\begin{array}{l}\text { Abkatum } \\
\text { «D» }\end{array}$ & $\begin{array}{l}\text { Productio } \\
\mathrm{n}\end{array}$ & Dodécapode & 46.90 & $1,2,3,5,6,7,9$ \\
\hline $\begin{array}{l}\text { Batab } \\
\text { «A» }\end{array}$ & $\begin{array}{l}\text { Perforatio } \\
\mathrm{n}\end{array}$ & Octopode & 25.30 & $1,2,3,5,6$ \\
\hline Caan $« A »$ & $\begin{array}{l}\text { Perforatio } \\
\mathrm{n}\end{array}$ & Octopode & 32.20 & $1,2,3,5,6$ \\
\hline Chuc $\ll B »$ & $\begin{array}{l}\text { Perforatio } \\
\mathrm{n}\end{array}$ & Octopode & 33.80 & $1,2,3,5,6,8,9,12$ \\
\hline Pool «A» & $\begin{array}{l}\text { Productio } \\
\mathrm{n}\end{array}$ & Octopode & 35.30 & $\begin{array}{l}1,2,3,4,5,6,7,9 \\
10,11,12\end{array}$ \\
\hline Pool «A» & Liaison & Tétrapode & 34.40 & $1,2,3,5,6,9,11$ \\
\hline Pool «A» & Habitation & Octopode & 33.80 & $1,2,3,5,6$ \\
\hline Pool «A» & $\begin{array}{l}\text { Compressi } \\
\text { on }\end{array}$ & Octopode & 33.80 & $\begin{array}{l}1,2,3,4,5,6,7,8,9, \\
12\end{array}$ \\
\hline Pool «A» & $\begin{array}{l}\text { Perforatio } \\
\mathrm{n}\end{array}$ & Octopode & 33.80 & $1,2,3,4,5,6,9,12$ \\
\hline Pool «D» & $\begin{array}{l}\text { Perforatio } \\
\mathrm{n}\end{array}$ & Octopode & 38.90 & $1,2,3,5,6,8,9$ \\
\hline $\begin{array}{l}\text { Uech } \\
\text { «A» }\end{array}$ & $\begin{array}{l}\text { Perforatio } \\
\mathrm{n}\end{array}$ & Octopode & 51.00 & $1,2,3,5,6,7$ \\
\hline
\end{tabular}

Table 3: Type d'endommagement présenté par les plates-formes dans la Région Pétrolière Sud-Ouest 


\section{Modèles hind cast utilisés par l'industrie pétrolière mexicaine}

Actuellement, diverses institutions au monde réalisent études pour le pronostic des effets des ouragans et des « Nords » avec un tel but on a développé modèles mathématiques pour la simulation des météores. Au moyen de ces modèles de simulation historique d'orage (hindcast), on prétend obtenir des cartes de valeurs minimales probables des principaux effets de ces orages en fonction de périodes de retour habituelles pour la conception de structures. Les premières installations marines au Mexique ont été conçus en utilisant de l'information météorologique et océanographiques obtenu à travers des modèles de hind cast. Ces modèles partent des méthodes empiriques et semi empiriques basées principalement dans les travaux de Bretschneider (OTC, 1972).

En 1995, la côte sud du Golfe du Mexique a été frappée par l'ouragan Roxanne. Cet orage a été considéré la plus grave du XX siècle dans la zone de plates-formes (Cardone y Ramos, 1998), et il a été prés des intensités de l'orage de conception des installations marines.

\section{L'exploitation pétrolière en eaux profondes}

Le prochain défi de l'industrie Pétrolière Mexicaine est la exploitation des hydrocarbures en eaux profondes, au-delà de $500 \mathrm{~m}$ et jusqu'à $2500 \mathrm{~m}$. Ceci représentera que pour l'année 2010, 1.6\% de la production totale des hydrocarbures au Mexique (60 millions de barils par jour) proviendra des installations pétrolières en eaux profondes. Jusqu'à présent, on a identifié dans les eaux profondes du Golfe du Mexique 170 opportunités d'exploration et approuvé deux prospectus, (voir figure 3 ).

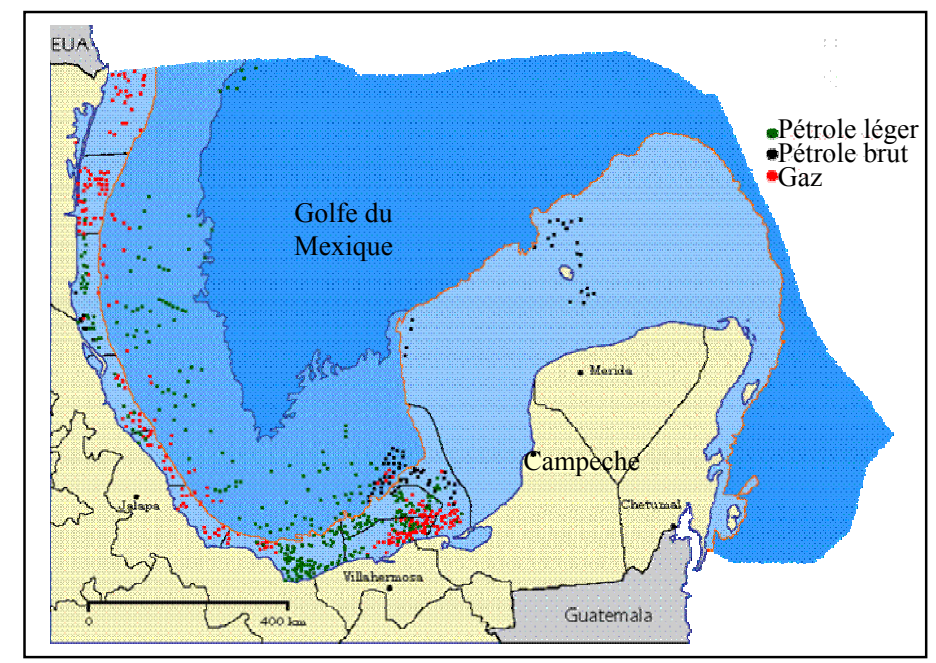

Figure 3: Opportunités d'exploration au Golfe du Mexique

\section{Conclusions}

Depuis l'expropriation pétrolière au Mexique durant l'année 1938, Pemex a le contrôle de l'exploitation d'hydrocarbures dans le pays. Jusqu'au 1965 commencent les activités pétrolières offshore au Mexique. Actuellement $82 \%$ de la production totale d'hydrocarbures dans le pays provient de la région marine (approximativement 2.5 millions de barils par jour). Pemex dispose de nos jours plus de 220 plates-formes pétrolières et plus de $2,400 \mathrm{~km}$ de lignes sous-marines. 
Un des défis de l'industrie pétrolière nationale est l'exploitation des hydrocarbures dans des eaux profondes, au-delà de $300 \mathrm{~m}$ et jusqu'au 2,000 $\mathrm{m}$ dans une première étape.

Pour l'année 2010, 1.6\% de la production d'hydrocarbures au Mexique (80,000 barils par jour) proviendra des installations pétrolières dans des eaux profondes. Pour faire face à ce défi, Pemex a entamé l'étude et le développement de nouvelles techniques d'exploitation des hydrocarbures à de grandes profondeurs

\section{Références}

1.Pemex Exploracion y Producción, (1999). Las reservas de hidrocarburos de Mexico. Petroleos Mexicanos, volumen 1, Mexico, 193p.

2.Pemex, (1979). La ruta del petroleo. La Exploracion petrolera en Mexico. Nosotros los petroleros, Mexico, p.15-18

3.Torres Martínez H., (1998). Evaluacion estructural de plataformas fijas con un criterio propio para la Sonda de Campeche. Tesis de licenciatura, Universidad La Salle, México, 185 p.

4.Soriano Martinez, J. A., (1999). Caracterizacion de peligros oceanograficos y meteorologicos. Primer curso de aplicación del criterio transitorio: Plataformas fijas. Instituto Mexicano del Petroleo, Division Plataformas, Mexico, 17p.

5.Bretschneider, Ch. (1972). A non-dimensional stationary hurricane wave model. University of Hawai, 1517, Offshore Technology Conference.

6.Cardone, V., Ramos R., (1998). Wave, wind and current characteristics of the Bay of Campeche. 9687 Offshore Tecnology Conference.

7.Pemex Exploracion y Produccion, (2002). Evaluacion del potencial de agues profundas en el Golfo de Mexico, Reporte técnico, 54 p. 